\title{
Mit neuen Publikationsstandards auf der Höhe der Zeit
}

SÄZ-Verlagsleitung

Korrespondenz:

Redaktion

Schweizerische Ärztezeitung

Farnsburgerstrasse 8

CH-4132 Muttenz

redaktion.saez[at]emh.ch

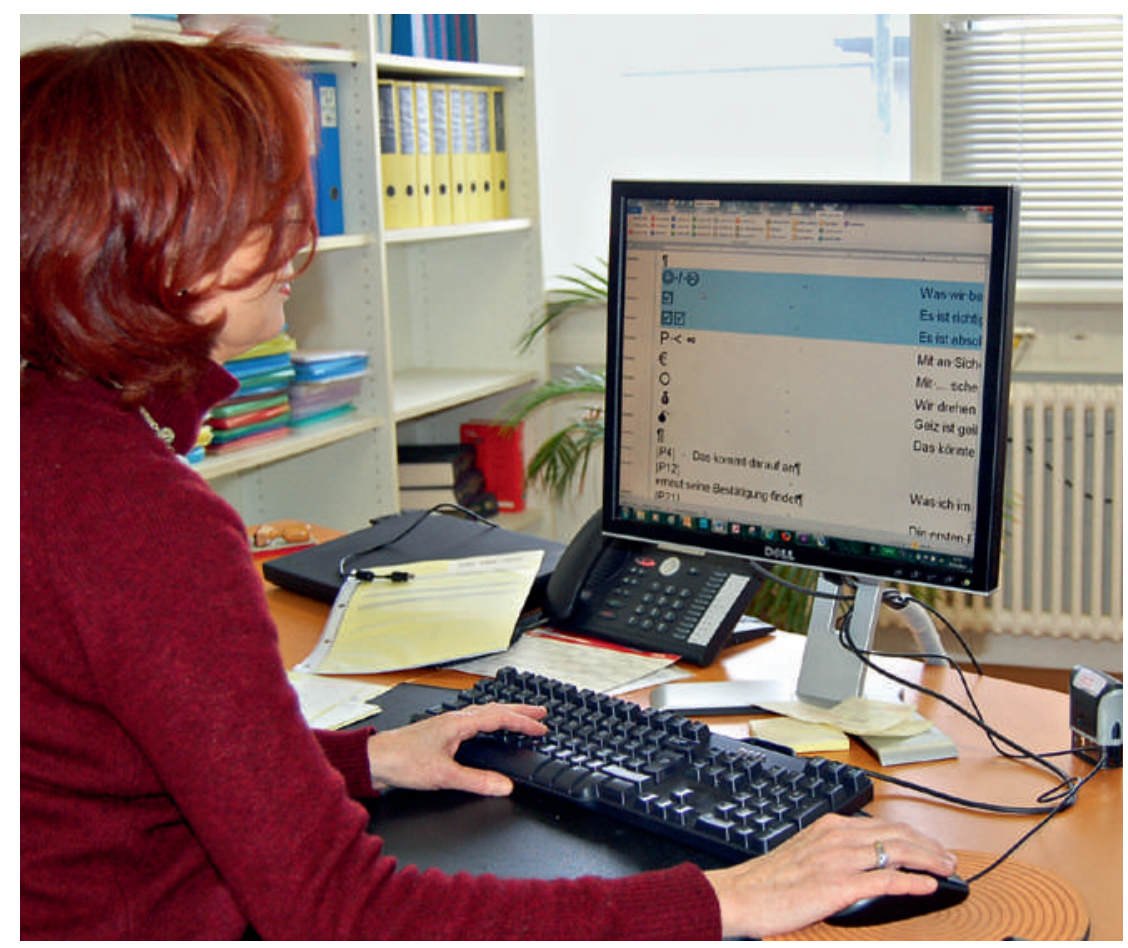

Die Managing Editorin der Ärztezeitung ist begeistert von der neuen Software: Das Seitenbudget kann besser eingehalten werden, ohne den Lesern wesentliche Informationen vorzuenthalten. geschätzte Autorinnen und Autoren der Schweizerischen Ärztezeitung

Rückläufige Einnahmen aus der Schaltung von Inseraten und steigende Produktionskosten haben uns in der Vergangenheit zu unpopulären Massnahmen ver-
Liebe Leserinnen und Leser, anlasst. Unter anderem mussten wir den Umfang der in der Printausgabe veröffentlichten Beiträge schrittweise reduzieren. Um weiteren Abstrichen an der Artikellänge zulasten des Inhalts vorzubeugen, sollen in Zukunft die Artikel durch einen zeitgemässen Kommunikationsstil effizienter gestaltet werden. Die Devise lautet: «Mehr Inhalt auf weniger Raum.»
Unsere neue Publikations-Software Med-XChange $^{\odot}$ erlaubt es, die eingereichten Beiträge automatisch auf heute übliche Kommunikationsformen im Klinik- oder Praxisalltag bzw. in den sozialen Medien zu übertragen. Mit der Artikeltransformation sind Textkürzungen ohne Qualitätseinbussen möglich. Darüber hinaus wird der Schreibstil mit der Auf-

\section{Mit der Artikeltransformation sind Textkürzungen ohne Qualitäts- einbussen möglich.}

nahme von umgangssprachlichen Gepflogenheiten und modernen Ausdrucksformen eine natürliche Frische erhalten. Nachfolgend möchten wir Sie auf die wichtigsten Änderungen aufmerksam machen, die sich während der Testphase an einer nicht repräsentativen Stichprobe bewährt haben:

Medizinische Fachausdrücke werden durch standardmässig verwendete Abkürzungen und Akronyme ersetzt. Entsprechende Satzkürzungen und die Vermeidung von Fremdwörtern verbessern den Lesefluss. Das Kennenlernen neuer Abkürzungen fördert zudem die Kommunikation im Alltag. In der Regel sind die Abkürzungen bzw. die Akronyme eindeutig (vgl. Tab. 1); wenn nicht, ist die daraus resultierende Verunsicherung wahrscheinlich auch nicht grösser als die Irritationen durch unverständliche Fachausdrücke. Um Missverständnisse zu vermeiden, sollten Sie in Ihrem ureigenen Interesse - das korrekte Verständnis einer Aussage immer aus dem inhaltlichen Zusammenhang ableiten.

\section{Tabelle 1}

Ausgewählte medizinische Abkürzungen und Akronyme.

\begin{tabular}{ll}
\hline AP & Alkalische Phosphatase; Anita Pectoris \\
\hline EZ & Ernährungszustand; eineiige Zwillinge \\
\hline $\mathrm{EZ}^{2}$ & Ernährungszustand von eineiigen Zwillingen \\
\hline HWI & Harnwegsinfekt; Hinterwandinfarkt \\
\hline LTx & Lebertransplantation; Lungentransplantation \\
\hline p. a. & peranal; posterior anterior \\
\hline p.o. & peroral; post ovulationem \\
\hline VSD & Ventrikelseptumdefekt; Vorhofseptumdefekt \\
\hline
\end{tabular}


Die persönliche Meinung der Autoren lässt sich oftmals besser durch Symbole und Piktogramme darstellen. Beispiele sind in Tabelle 2 zu finden. Vor allem können Emotionen anschaulich zum Ausdruck gebracht werden - ohne viele Worte machen zu müssen und dabei Gefahr zu laufen, sich im Ton zu vergreifen. Die Artikel gewinnen mit dieser Art der Darstellung zudem an Variationsbreite und bringen Abwechslung für unsere Leserinnen und Leser. Trotz mehrfacher Anregung seitens unserer jüngeren Kolleginnen und Kollegen werden wir von Inflektiven («seufz», «gähn») allerdings nur sehr sparsamen Gebrauch machen.

\section{Nichtssagende Standardformulierungen können so auf einen angemessenen Umfang reduziert werden.}

Die 50 am häufigsten verwendeten Phrasen mit eingeschränkter Aussagekraft werden durch Platzhalter ersetzt ([P1] bis [P50]). Beiträge mit vielen nichtssagenden Standardformulierungen können somit auf einen angemessenen Umfang reduziert werden. Die Anzahl von Platzhaltern [P...] in einem Artikel hilft den Leserinnen und Lesern ausserdem dabei, sich rasch eine Meinung über die Originalität eines Beitrags zu bilden. Um der Kreativität einzelner Autoren zu begegnen, wird die Liste fortwährend ergänzt. In der Tabelle 3 finden Sie einige ausgewählte Phrasen und Standardformulierungen aus den aktuellen «Top 50».

In den nächsten Ausgaben der Schweizerischen Ärztezeitung werden wir die überarbeiteten Autorenrichtlinien publizieren und Sie mit den zukünfti- gen Abkürzungen, Symbolen und Phrasen-Platzhaltern vertraut machen. Wir sind überzeugt, dass die Mehrheit der schweizerischen Ärzteschaft (-) und [P24] glaubt. Und $\square$, wenn Sie meinen, dass [P43].

\section{Tabelle 2}

Ausgewählte Symbole und Piktogramme.

(:) / (:) Was wir befürworten / nicht befürworten

$\square \quad$ Es ist richtig, dass ...

$\square \square \quad$ Es ist absolut richtig, dass ...

$\mathrm{P}<\infty \quad$ Mit an Sicherheit grenzender Wahrscheinlichkeit

$€ \quad$ Mit ... scheint es unaufhaltsam bergab zu gehen.

O Wir drehen uns im Kreis.

ङ Geiz ist geil.

* Das könnte ernsthafte Konsequenzen nach sich ziehen.

\section{Tabelle 3}

Ausgewählte Phrasen und Standardformulierungen.

[P4] Das kommt darauf an.

[P12] Was ich immer schon behauptet habe und hier erneut seine Bestätigung findet.

[P21] Die ersten Resultate sind vielversprechend, aber weitere Untersuchungen sind erforderlich.

[P24] Ein erster Schritt in die richtige Richtung.

[P26] Trotz all meiner Verdienste habe ich nicht die Bodenhaftung verloren.

[P29] Zum damaligen Zeitpunkt war das die richtige Entscheidung (oder: «lch war jung und brauchte das Geld»).

[P31] Wir werden mit Mut und Offenheit die Probleme angehen und einen tragfähigen Kompromiss anstreben.

[P43] Das soll wohl wieder so ein Aprilscherz sein. (-) 